\title{
Transitivity and invariant measures for the geometric model of the Lorenz equations
}

\author{
CLARK ROBINSON \\ Department of Mathematics, Northwestern University, Evanston, IL 60201, USA
}

(Received 12 October 1983 and revised 16 January 1984)

\begin{abstract}
This paper concerns perturbations of the geometric model of the Lorenz equations and their associated one-dimensional Poincaré maps. R. Williams has shown that if a map of the interval of the type arising from the Lorenz equations satisfies $f^{\prime}(x)>2^{\frac{1}{2}}$ everywhere except at the discontinuity then $f$ is locally eventually onto (1.e.o.) and so topologically transitive [14]. We show roughly that if $A^{ \pm}$are the end points of the interval, and their iterates $f^{j}\left(A^{ \pm}\right)$stay on the same side of the point of discontinuity for $0 \leq j \leq k$, and $f^{\prime}(x)>2^{1 /(k+1)}$ everywhere, then $f$ is l.e.o. Secondly, we show that the one-dimensional Poincare map of any $C^{r}$ perturbation of the geometric model (for large enough $r$ ) has an ergodic measure which is equivalent to Lebesgue measure. This result follows by showing it is $C^{1+\alpha}$ and satisfies a theorem of Keller, Wong, Lasota, Li \& Yorke.
\end{abstract}

\section{Statement of results}

The Lorenz equations are

$$
\begin{aligned}
& \dot{x}=-\sigma x+\sigma y \\
& \dot{y}=\rho x-y+x z \\
& \dot{z}=-\beta z+x y .
\end{aligned}
$$

When the parameter values are $\sigma=10, \beta=8 / 3$, and $\rho=28$, numerical integration indicates these equations have a strange attractor. Since the analytic estimates have not been made to prove the strange attractor exists, a geometric model was introduced which has a strange attractor of the type indicated by the numerical integrations. See [4], [5], or [13] for a description of this geometric model.

The important aspect of the model for this paper is the form of the Poincare return map for the flow $\psi$ from a transversal contained in $\{z=1\}$ to itself; it is assumed to be of the form

$$
P_{\psi}(x, y)=\left(f_{\psi}(x), g_{\psi}(x, y)\right)
$$

where $g_{\psi}(x, \cdot)$ is a contraction and $f=f_{\psi}: I \equiv\left[A^{-}, A^{+}\right] \rightarrow I$ satisfies the following conditions:

(1.2) (a) $f$ has a single discontinuity at some $x=C$;

(b) the limit of $f$ from the left side of $C$ is $A^{+}, f(C-0)=A^{+}$, and the limit from the right side of $C$ is $A^{-}, f(C+0)=A^{-}$, and $f\left(A^{-}\right)<C<f\left(A^{+}\right)$; 
(c) $f$ is non-uniformly continuously differentiable on $\left[A^{-}, A^{+}\right]-\{C\}$ and there is a $\lambda>1$ such that $f^{\prime}(x)>\lambda$ for all $x \neq C$;

(d) the limit of $f^{\prime}(x)$ is infinity as $x$ approaches $C$ from either side.

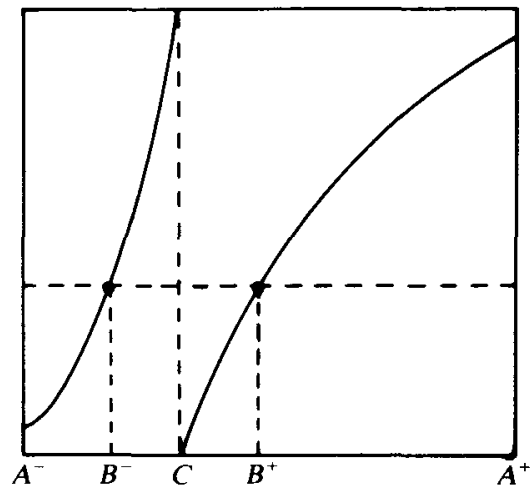

FIGURE 1

For our theorems we need two more conditions for which we need some more definitions. Assume $f$ satisfies $1.2\left(\right.$ a) and let $B^{ \pm}$be such that $f\left(B^{ \pm}\right)=C$ with $A^{-}<B^{-}<C<B^{+}<A^{+}$. Let $M\left(f^{\prime}\right)(x)$ be the mean value of $f^{\prime}$ from $C$ to $x$ (with either $x<C$ or $x>C$ ):

$$
M\left(f^{\prime}\right)(x)=\frac{1}{x-C} \int_{C}^{x} f^{\prime}(t) d t=\frac{f(x)-f(C)}{x-C} .
$$

As usual, a function $g$ is said to be $C^{r+\alpha}$ with $0<\alpha \leq 1$ if it is $C^{r}$ and the $r$ th derivative, $D^{r} g(p)$, satisfies a Hölder condition with exponent $\alpha$. The last two conditions on $f: I \equiv\left[A^{-}, A^{+}\right] \rightarrow I$ are as follows:

(e) let $k$ be such that

$$
\begin{array}{ll}
f^{j}\left(A^{-}\right) \leq C & \text { for } 0 \leq j \leq k \\
f^{j}\left(A^{+}\right) \geq C & \text { for } 0 \leq j \leq k,
\end{array}
$$

$M\left(f^{\prime}\right)(x) \geq \Lambda$ for $B^{-} \leq x \leq B^{+}$where $f\left(B^{ \pm}\right)=C, f^{\prime}(x) \geq \lambda>1$ for all $x \neq C$, and $\Lambda \lambda^{k}>2$;

(f) the inverse of $f$ extends to a $C^{1+\alpha}$ function for some $\alpha>0$ on both $J_{1}=\left[f\left(A^{-}\right), A^{+}\right]$and $J_{2}=\left[A^{-}, f\left(A^{+}\right)\right] ; \quad g_{1}: J_{1} \rightarrow I_{1} \subseteq\left[A^{-}, A^{+}\right]$and $g_{2}: J_{2} \rightarrow I_{2} \subseteq$ $\left[A^{-}, A^{+}\right]$are $C^{1+\alpha}$.

Below we discuss how condition 1.2(f) implies that $f_{\psi}$ has an invariant measure for any perturbation $\psi$. First, we discuss the connection of $1.2(\mathrm{e})$ with the topological transitivity of $f_{\psi}$. A map $f:\left[A^{-}, A^{+}\right] \rightarrow\left[A^{-}, A^{+}\right]$is said to be topologically transitive if there is a point $A^{-} \leq x \leq A^{+}$with the $\omega$-limit set of $x$ equal to the whole interval $\left[A^{-}, A^{+}\right], \omega(x)=\left[A^{-}, A^{+}\right]$. The map $f$ is said to be locally eventually onto (1.e.o.) if every open interval $J$ in $\left[A^{-}, A^{+}\right]$has some iterate $k$ such that $f^{k}(J)=\left[A^{-}, A^{+}\right]$. By a theorem of Birkhoff if $f$ is l.e.o. then a residual set of points have $\omega(x)=\left[A^{-}, A^{+}\right]$ and so $f$ is certainly topologically transitive. 
R. Williams showed that if $f$ satisfies $1.2(\mathrm{a})-(\mathrm{c})$ and $f^{\prime}(x)>2^{\frac{1}{2}}$ for all $x \neq C$, then $f$ is l.e.o. [14]. The difficulty is that computer integration of the Lorenz equations (1.1) indicate that $f^{\prime}(x)$ is near 1 for $x$ near the end points so the assumption does not hold. The next theorem gives the same result with weaker hypotheses which seem verifiable.

TheOREM A. Let $f:\left[A^{-}, A^{+}\right] \rightarrow\left[A^{-}, A^{+}\right]$be a map which satisfies $1.2(a)-(c)$ and 1.2(e) with $k \geq 2$. Then $f$ is l.e.o.

The proof of this theorem is contained in $\$ 2$ and uses some ideas from the proof of R. Williams. It should be remarked that $C$. Sparrow found examples of maps satisfying 1.2(a)-(c) which are not 1.e.o. [13]. R. Williams showed me the following example. Let the subintervals $I_{j}^{ \pm}$be as in figure 2 and let their images be as follows:

$$
\begin{array}{ll}
f\left(I_{1}^{-}\right)=I_{2}^{-}, & f\left(I_{2}^{-}\right)=I_{3}^{-} \cup I_{3}^{+}, \quad f\left(I_{3}^{-}\right)=I_{1}^{+}, \\
f\left(I_{3}^{+}\right)=I_{1}^{-}, & f\left(I_{1}^{+}\right)=I_{2}^{+}, \quad f\left(I_{2}^{+}\right)=I_{3}^{-} \cup I_{3}^{+} .
\end{array}
$$

Clearly $I_{1}^{-} \cup I_{2}^{-} \cup I_{3}^{-} \cup I_{3}^{+} \cup I_{2}^{+} \cup I_{1}^{+}$is invariant so $f$ is not l.e.o. In terms of $1.2(\mathrm{e})$, $k=2$. The third iterate of $I_{1}^{-}, f^{3}\left(I_{1}^{-}\right)=I_{1}^{-} \cup I_{1}^{+}$so $\Lambda \lambda^{2} \leq 2$ but there is no strict

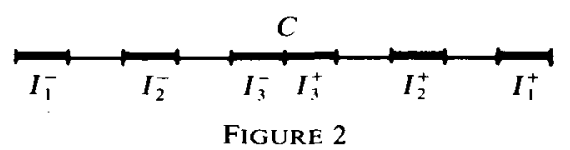

inequality. On these sub-intervals, in some sense $\Lambda \lambda^{2}=2$ so it is the first place where the hypothesis of theorem A does not apply.

R. Williams (results amended by $\mathrm{C}$. Sparrow) has given conditions on the kneading sequences (i.e. the sequences of sides on which $f^{j}\left(A^{-}\right)$and $f^{j}\left(A^{+}\right)$lie) which they claim imply $f$ is 1.e.o. See [13]. The disadvantage with using kneading invariants to show a specific example is l.e.o. is that in general the whole infinite sequences of iterates of the end points must be determined. The above theorem uses a finite amount of the kneading invariant together with an assumption on the derivative.

Turning to the existence of an invariant measure, there is the following result.

TheOrem B. Assume the geometric model of the Lorenz equations has eigenvalues at the fixed point (and thus the Liapunov exponents on the strange attractor) $a,-b$, and $-c$ where $0<c<a<b, b-c-(1+\alpha) a>0$ for some $\alpha>0$, and $a / c>1+\alpha$. Moreover assume the eigenvalues at the fixed point satisfy non-resonance conditions of order $r$ where $r$ is large enough to $C^{2}$ linearize near the origin. (For the eigenvalues of (1.1) with the values of the parameters given above, $r=20$ is needed.) Then there is an open set $N$ in $C^{r}$ flows which are $C^{2}$ near the model equations such that for $\psi$ in $N$ the associated Poincaré map of the interval, $f_{\psi}:\left[A_{\psi}^{-}, A_{\psi}^{+}\right] \varsigma$, has an invariant measure $h d m$ where $m$ is Lebesgue measure and $h$ is an $L^{1}$ density function. Further,

(i) there is a finite union of closed intervals $L$ such that support $h=L$;

(ii) $h d m$ is equivalent to Lebesgue on $L(h(x)>0$ a.e.); and

(iii) $f_{\psi}$ is ergodic with respect to $h d m$. 
If the geometric model also satisfies the assumption of theorem A (or if $\psi$ is l.e.o.), then support $h=\left[A^{-}, A^{+}\right]$.

The eigenvalue assumption implies there is an open set of flows satisfying condition (1.2) as discussed in $\S 3$. The existence of an invariant measure $h d m$ follows from a result of G. Keller [7] and S. Wong [16] which shows the existence of an absolutely continuous measure for a class of functions possessing discontinuities which include functions satisfying 1.2(a)-(d), (f). S. Wong uses an extra condition not needed by G. Keller to prove the result. We indicate a slight change in his proof which eliminates this extra condition in $\S 4$. The support of the measure follows from a theorem of T. Li \& J. Yorke [9] as discussed in [15]. Moreover $h(x)>0$ for almost all $x$ in the support $L$ so $h d m$ is equivalent to Lebesgue measure on $L$. If $f$ is l.e.o. and has a single discontinuity, then a consequence of their result is that the support of $h$ is the whole interval $\left[\mathrm{A}^{-}, A^{+}\right]$as stated in the theorem, $L=\left[A^{-}, A^{+}\right]$.

A related result was proved by L. A. Bunimovich \& Y. G. Sinai, [3] and [12]. They studied the two-dimensional Poincaré map $P(x, y)$ and showed that if it satisfied hyperbolicity conditions then it possesses a Bowen-Ruelle-Sinai invariant measure, [3] and [12]. The quotient of this measure by projection along the stable foliation induces a measure on the interval but it is not clear that it has the properties given above. In any case, the proof here uses a different approach from theirs. Also, W. Parry studied piecewise linear maps which are topologically conjugate to the maps of the interval arising from the Lorenz equations and showed that they have an absolutely continuous measure [10]. The topological conjugacy does not carry over this measure without some smoothness, so our result does not follow directly from his.

\section{Proof of theorem A}

Let $L(J)$ be the length of any subinterval $J$. Let $I=\left[A^{-}, A^{+}\right]$and $B^{ \pm}$be such that $f\left(B^{ \pm}\right)=C$. Take any interval $J(0) \subset I$. We need to show there is an $n$ such that $f^{n}(J(0))=I$. Assume $J(i)$ is defined. Let $J^{\prime}(i)$ be the longer component of $J(i)-\{C\}$. In particular if $C$ is not in $J(i)$ then $J^{\prime}(i)=J(i)$. Let $J(i+1)=f\left(J^{\prime}(i)\right)$. If $C$ is not in $J(i)$ then $L(J(i+1)) \geq \lambda L(J(i))$. Since $I$ is finite, there is a subsequence $i_{j}$ such that $C$ is in $J\left(i_{j}-1\right)$. Thus $J\left(i_{j}\right)=\left[A^{-}, x_{j}\right]$ or $\left[x_{j}, A^{+}\right]$. The idea of the proof is that $J\left(i_{j}+p\right)$ does not contain $C$ for $0 \leq p \leq k-1$ unless $J(i)$ contains $\left[B^{-}, C\right]$ or [C, $\left.B^{+}\right]$for some $i_{j} \leq i<i_{j}+k$. See lemma 2 below. Thus except in the latter case $L\left(J\left(i_{j}+k\right)\right) \geq \lambda^{k} L\left(J\left(i_{j}\right)\right)$ and cannot get cut until at least the $k+1$ st iterate. It follows that

$$
L\left(J\left(i_{j+1}\right)\right) \geq \frac{1}{2} \Lambda \lambda^{k} L\left(J\left(i_{j}\right)\right),
$$

and the length of the intervals $J\left(i_{j}\right)$ grows until $J(i)$ contains $\left[B^{-}, C\right]$ or $\left[C, B^{+}\right]$for some $i_{j} \leq i<i_{j+1}$. Lemma 1 shows that $f^{3}(J(i)) \supset\left[A^{-}, A^{+}\right]$. We proceed with the details.

LEMMA 1. If $J(i) \supset\left[B^{-}, C\right]$ or $\left[C, B^{+}\right]$then $f^{3}(J(i)) \supset\left[A^{-}, A^{+}\right]=I$ so the $(i+3) r d$ iterate of $J(0)$ covers $I$. 
Proof. Assume the first case. The other is similar. Then $f(J(i)) \supset\left[C, A^{+}\right]$, $f^{2}(J(i)) \supset\left[A^{-}, C\right] \cup\left[C, B^{+}\right]$since $k \geq 2$, so

$$
f^{3}(J(i)) \supset f\left(\left[A^{-}, C\right]\right) \cup f\left(\left[C, B^{+}\right]\right) \supset\left[C, A^{+}\right] \cup\left[A^{-}, C\right]=I .
$$

LEMMA 2. For the subsequence $J\left(i_{j}\right)=\left[A^{-}, x_{j}\right]$ or $\left[x_{j}, A^{+}\right]$either

$$
L\left(J\left(i_{j+1}\right)\right) \geq \frac{1}{2} \Lambda \lambda^{k} L\left(J\left(i_{j}\right)\right) \text { or } f^{3}(J(i))=I
$$

for some $i_{j} \leq i<i_{j+1}$.

Proof. Take the case $J\left(i_{j}\right)=\left[A^{-}, x_{j}\right]$. If $f^{p}\left(x_{j}\right) \geq C$ for some $0 \leq p \leq k-1$ then $J\left(i_{j}+p\right) \supset\left[B^{-}, C\right]$ since $f^{p}\left(A^{-}\right) \leq B^{-}$. By lemma $1, f^{3}\left(J\left(i_{j}+p\right)\right)=I$ and we are done. Thus for $0 \leq p \leq k-1$, we can assume $f^{p}\left(x_{j}\right)<C$, and so $J^{\prime}\left(i_{j}+p\right)=J\left(i_{j}+p\right)$ and $L\left(J\left(i_{j}+k\right)\right) \geq \lambda^{k} L\left(J\left(i_{j}\right)\right)$. Also $i_{j+1}-1 \geq i_{j}+k$. There are no cuts from $i_{j}+k$ to $i_{j+1}-1$ so

$$
L\left(J\left(i_{j+1}-1\right)\right) \geq L\left(J\left(i_{j}+k\right)\right) \geq \lambda^{k} L\left(J\left(i_{j}\right)\right)
$$

If $J^{\prime}\left(i_{j+1}-1\right)$ contains $\left[B^{-}, C\right]$ or $\left[C, B^{+}\right]$then we are done by lemma 1 again. Thus we can assume $J^{\prime}\left(i_{j+1}-1\right) \subset\left[B^{-}, C\right]$ or $\left[C, B^{+}\right]$so

$$
L\left(J\left(i_{j+1}\right)\right) \geq \Lambda L\left(J^{\prime}\left(i_{j+1}-1\right)\right) \geq \frac{1}{2} \Lambda L\left(J\left(i_{j+1}-1\right)\right) \geq \frac{1}{2} \Lambda \lambda^{k} L\left(J\left(i_{j}\right)\right) .
$$

This completes the proof.

By repeated application of lemma $2, L\left(J\left(i_{j}\right)\right) \geq\left(\Lambda \lambda^{k} / 2\right)^{j} L\left(J\left(i_{0}\right)\right)$ until $f^{3}(J(i))=I$ for some $i_{j} \leq i<i_{j+1}$. Because the lengths are growing geometrically and $L(I)$ is finite, eventually some iterate covers $I$. This completes the proof of theorem $A$.

\section{Openness of condition 1.2}

It was proved in [11] that there is an open set in the $C^{2}$ flows which satisfies conditions $1.2(\mathrm{a})-(\mathrm{c})$. The openness of condition $1.2(\mathrm{~d})$ in $C^{2}$ flows is also proved in [11], but an easier proof is obtained by a $C^{1}$ linearization of the flow near the fixed point as pointed out to me by $C$. Tresser. This $C^{1}$ linearization can be done if the flow is $C^{2}$, [2]. A straightforward argument (using 1.2(d)) shows there is an open set of $C^{2}$ flows which satisfy $1.2(\mathrm{e})$ for the same $k$. To get the openness of condition 1.2(f), the projection onto the branched manifold along the strong contracting direction must be $C^{1+\alpha}$, c.f. [11]. If the eigenvalues satisfy the conditions of theorem $\mathrm{B}$, then there is the usual proof as discussed in [11] using the $C^{1+\alpha}$ version of [6, theorem 6.1]. The only difficulty in obtaining $1.2(\mathrm{f})$ is thus in showing the branches of the inverse extend to the closures at $A^{+}$and $A^{-}$in such a manner so that the extension is $C^{1+\alpha}$. This can certainly be done by $C^{2}$ linearizing the flow. For the eigenvalues of (1.1) this requires $r=20$. It would be good to give a proof of this $C^{1+\alpha}$ extension assuming only $r=2+\alpha$. A possible proof would use the formula for the derivative of the Poincaré map of a flow of a $C^{1}$ vector field in two dimensions given in [1]. This formula can certainly be used to give an alternative proof of $1.2(\mathrm{~d})$.

\section{Modifications in the proof of Wong}

As stated in [16, remark 4, p. 513], the only place where the added assumptions are used is in the estimate of $\Delta_{21}$ on page 511 . (Of course this result in contained in [7, 
lemma 3.1].) Using the notation of that paper we prove the estimate on $\Delta_{2}=\Delta_{21}+\Delta_{22}$ without the added assumption. Here $\mathscr{R}=\left\{y_{j}\right\}, f$ is the density function, $\Phi=\tau^{N}$ for some $N$ is the transformation, $\psi_{i}$ are branches of the inverse, and $\sigma_{i}=\left|\psi_{i}^{\prime}\right| ; v_{p}(g ; J)$ is the $p$-variation of the function $g$ on the interval $J$.

$$
\begin{aligned}
\Delta_{2} & =\left\{\sum_{\mathscr{R}}\left[\sum_{i=1}^{u}\left|f \circ \psi_{i}\left(y_{j}\right)\right|\left|\sigma_{i}\left(y_{j}\right)-\sigma_{i}\left(y_{j-1}\right)\right|\right]^{p}\right\}^{1 / p} \\
& \leq \sum_{i=1}^{u} \sum_{\mathscr{R}}\left\{\left|f \circ \psi_{i}\left(y_{j}\right)\right|^{p}\left|\sigma_{i}\left(y_{j}\right)-\sigma_{i}\left(y_{j-1}\right)\right|^{p}\right\}^{1 / p} \\
& \leq \sum_{i=1}^{u} \sum_{\mathscr{R}}\left|f \circ \psi_{i}\left(y_{j}\right)\right| v_{p}\left(\sigma_{i} ;\left[y_{j-1}, y_{j}\right]\right) \\
& \leq \sum_{i=1}^{u}\left\{v_{p}\left(f ; I_{i}\right)+(1 / h) \int_{I_{i}}|f| d m\right\} \sum_{\mathscr{R}} v_{p}\left(\sigma_{i} ;\left[y_{j-1}, y_{j}\right]\right) \\
& \leq \sum_{i=1}^{u}\left\{v_{p}\left(f ; I_{i}\right)+(1 / h) \int_{I_{i}}|f| d m\right\} v_{p}\left(\sigma_{i} ; J_{i}\right) \\
& \leq\left\{\max _{i} v_{p}\left(\sigma_{i} ; J_{i}\right)\right\}\left\{v_{p}(f ; I)+(1 / h) \int|f| d m\right\} .
\end{aligned}
$$

This estimate does not contain a factor of a bound on the iterate of the constant function 1 or the bound on derivative of $\tau$, i.e. no $[M(N)]^{1 / q} t^{N / q}$. The rest of the proof is unchanged.

This work was partially supported by NSF Grant MCS-8301067.

\section{REFERENCES}

[1] A. Andronov et al. Theory of Bifurcation of Dynamical Systems on the Plane. Wiley: New York, 1973.

[2] G. R. Belickii. Functional equations and conjugacy of local diffeomorphisms of finite smooth class. Sov. Math. Dokl. 13 (1972), 56-59.

[3] L. A. Bunimovich \& Ya G. Sinai. Stochasticity of the attractor in the Lorenz model. In Nonlinear Waves, Proc. Winter School Gorki 1977, Nauka Moscow 1979, pp. 212-226.

[4] J. Guckenheimer \& P. Holmes. Non-linear Oscillations, Dynamical Systems and Bifurcation of Vector Fields. Springer-Verlag: New York-Heidelberg-Berlin, 1983.

[5] I. Guckenheimer \& R. Williams. Structural stability of Lorenz attractors. Publ. IHES 50 (1979), 59-72.

[6] M. Hirsch \& C. Pugh. Stable manifolds and hyperbolic sets. In Proc. Symp. Pure Math. 14 (1970), Amer. Math. Soc. pp. 133-163.

[7] G. Keller. Generalized bounded variation and applications to piecewise monotonic transformations. Preprint, University of Heidelberg, 1981.

[8] A. Lasota \& J. Yorke, On the existence of invariant measures for piecewise monotonic transformations. Trans. Amer. Math. Soc. 186 (1973), 481-488.

[9] T. Li \& J. Yorke. Ergodic transformations from an interval into itself. Trans. Amer. Math. Soc. 235 (1978), 183-192.

[10] W. Parry. The Lorenz attractor and related population model. In Ergodic Theory Conference Oberwolfach (edited by M. Denker and K. Jacobs) Lecture Notes in Math. 729 (1979). SpringerVerlag: New York, pp. 169-187.

[11] C. Robinson. Differentiability of the stable foliations for the model Lorenz equations. In Dynamical Systems and Turbulence (edited by D. Rand and L. S. Young) Lecture Notes in Math. 898 (1981). Springer-Verlag: New York, pp. 302-315.

[12] Ya G. Sinai. Hyperbolic conditions for Lorenz model. Phys. D 2 (1981), 3-7. 
[13] C. Sparrow. The Lorenz Equations: Bifurcation, Chaos and Strange Attractors. Applied Math. Sci. 41. Springer-Verlag: New York-Heidelberg-Berlin, 1982.

[14] R. Williams. The structure of Lorenz attractors. Publ. IHES 50 (1979), 73-99.

[15] S. Wong. Some metric properties of piecewise monotonic mappings of the unit interval. Trans. Amer. Math. Soc. 246 (1978), 493-500.

[16] S. Wong. Hölder continuous derivatives and ergodic theory, London Math. Soc. 22 (1980), 506-520. 